\title{
Article \\ Genome-Wide Identification, Characterization, and Expression Analysis of DDE_Tnp_4 Family Genes in Eriocheir sinensis
}

\author{
Yuanfeng $\mathrm{Xu}^{1}{ }^{1}$, Jinbin Zheng ${ }^{1}$, Yanan Yang ${ }^{1, *}$ and Zhaoxia Cui ${ }^{1,2}$ \\ 1 School of Marine Sciences, Ningbo University, Ningbo 315020, China; 1901130057@nbu.edu.cn (Y.X.); \\ zhengjinbin@nbu.edu.cn (J.Z.); cuizhaoxiao@nbu.edu.cn (Z.C.) \\ 2 Laboratory for Marine Biology and Biotechnology, Qingdao National Laboratory for Marine Science and \\ Technology, Qingdao 266071, China \\ * Correspondence: yangyanan@nbu.edu.cn; Tel.: +86-185-5902-3327
}

check for updates

Citation: Xu, Y.; Zheng, J.; Yang, Y.; Cui, Z. Genome-Wide Identification, Characterization, and Expression Analysis of DDE_Tnp_4 Family Genes in Eriocheir sinensis. Antibiotics 2021, 10, 1430. https://doi.org/ 10.3390/antibiotics10121430

Academic Editor: Juraj Gregáň

Received: 20 October 2021

Accepted: 19 November 2021

Published: 23 November 2021

Publisher's Note: MDPI stays neutral with regard to jurisdictional claims in published maps and institutional affiliations.

Copyright: (c) 2021 by the authors. Licensee MDPI, Basel, Switzerland. This article is an open access article distributed under the terms and conditions of the Creative Commons Attribution (CC BY) license (https:// creativecommons.org/licenses/by/ $4.0 /)$.

\begin{abstract}
DDE transposase 4 (DDE_Tnp_4) family is a large endonuclease family involved in a wide variety of biological processes. However, little information is available about this family in crustaceans. In this study, we used HMMER to identify 39 DDE_Tnp_4 family genes in Eriocheir sinensis genome, and the genes were classified into four subfamilies according to phylogenetic analysis. Gene expansions occurred among E. sinensis genome, and synteny analysis revealed that some DDE_Tnp_4 family genes were caused by tandem duplication. In addition, the expression profiles of DDE_Tnp_4 family genes in E. sinensis indicated that subfamily I and II genes were upregulated in response to acute high salinity and air exposure stress. E. sinensis is a kind of economical crustacean with strong tolerance to environmental stress. We confirmed the expansion of DDE_Tnp_4 family genes in E. sinensis and speculated that this expansion is associated with strong tolerance of E. sinensis. This study sheds light on characterizations and expression profiles of DDE_Tnp_4 family genes in E. sinensis and provides an integrated framework for further investigation on environmental adaptive functions of DDE_Tnp_4 family in crustaceans.
\end{abstract}

Keywords: DDE_Tnp_4 family; Eriocheir sinensis; gene expansion; environmental stress

\section{Introduction}

The Chinese mitten crab, Eriocheir sinensis, is one of the most commercially important crustaceans [1]. The life history of E. sinensis is more complicated than other economic crabs due to a special breeding migration. Adult E. sinensis mostly live in freshwater areas far from estuaries [2]. During breeding season, E. sinensis migrates downstream from fresh to brackish water, where they suffer complicated stress [3]. E. sinensis is a euryhaline species and a strong osmoregulator as they can function equally well in freshwater or brackish environments [4]. In addition, E. sinensis is a species capable of aerial respiration and can survive for extended periods of time, even days, without water [5]. Compared with Scylla paramamosain and Portunus trituberculatus, which are also commercially important crustaceans in China, E. sinensis exhibits enhanced tolerance to abiotic factors, including high salinity and air exposure [2,5-7]. Existing studies have revealed that strong tolerance to high salinity and air exposure of E. sinensis is linked to genes participating in innate immune system and many other physiological activities [8,9], such as Toll-like receptor (TLR), $\mathrm{Na}^{+}-\mathrm{K}^{+}$-ATPase, heat shock proteins (like Hsp70 and Hsp90), and superoxide dismutase (SOD) [10-12].

Furthermore, gene expansion has been associated with environmental adaptation [13]. In Chlamys farreri, the expansive $\mathrm{Cu} / \mathrm{Zn}$ SOD family genes are considered to assist in protecting the body against paralytic shellfish toxins (PSTs) [14]. The expansive subfamilies $\mathrm{G}$ and $\mathrm{H}$ of ATP-binding cassette (ABC) family in Daphnia pulex are thought to be critical in pollutant efflux and cell defense activities [15]. However, whether excellent abiotic stress tolerance of E. sinensis is linked to some expansive genes in E. sinensis remains unknown. 
DDE superfamily is a huge endonuclease family characterized by the presence of three conserved acidic residues (Asp-Asp-Glu) in their RNase H-like domain (RNH) active sites that can bind to magnesium ion and catalyze phosphodiester bond hydrolysis [16,17]. DDE transposase 4 (DDE_Tnp_4) family, belonging to DDE superfamily, is required for efficient DNA transposition. Some DDE_Tnp_4 family genes function as transcription factors, regulating the expression of diverse sets of genes implicated in immune regulation, angiogenesis, cell cycle regulation, stem cell pluripotency, and epigenetic gene silencing [18-20].

DDE_Tnp_4 family genes have been reported to expand during the evolution of some species, providing them with some evolutionary advantages. One member of DDE_Tnp_4 family is costly and exhibits evolutionary conservation patterns in coelacanth, Latimeria chalumnae genome, which benefits vertebrate invasion of terrestrial environment [21]. Several DDE_Tnp_4 family genes have undergone differential patterns of lineage-specific expansion in Acyrthosiphon pisum, implying that these genes might have a significant role in the diversification of morphology and adaptations [22].

This study was conducted to identify whether E. sinensis DDE_Tnp_4 family has expanded and whether this family is linked to stress tolerance of E. sinensis. We first identified and compared DDE_Tnp_4 family genes among different genomes in crustaceans and then investigated the localization of DDE_Tnp_4 family genes on E. sinensis chromosomes. Transcriptome data were employed to analyze the expression changes of E. sinensis DDE_Tnp_4 family genes under high salinity and air exposure stress. This study provides the basic information and clues for further investigation of the environmental adaptive function of DDE_Tnp_4 family.

\section{Results}

\subsection{The Expansion of DDE_Tnp_4 Family}

Herein, 39, 16, 3, and 4 DDE_Tnp_4 family genes were identified in E. sinensis, S. paramamosain, P. trituberculatus, and D. pulex, respectively. Meanwhile, the number of DDE_Tnp_4 family genes accounted for $0.1391 \%, 0.0872 \%, 0.0072 \%$, and $0.0131 \%$ of whole-genome protein-coding genes in E. sinensis, S. paramamosain, P. trituberculatus, and D. pulex, respectively (Amino acid sequences can be found in Sequence S1 in Supplementary Materials). The computational analysis of gene family evolution (CAFE) output result indicated that, among the three Brachyura species tested, DDE_Tnp_4 family genes of E. sinensis branch expanded significantly $\left(p=1.97756 \times 10^{-6}\right)$ compared with Brachyura ancestor species (Figure 1), with a total of 20 expansions. This result indicated that DDE_Tnp_4 family expanded among E. sinensis genome.

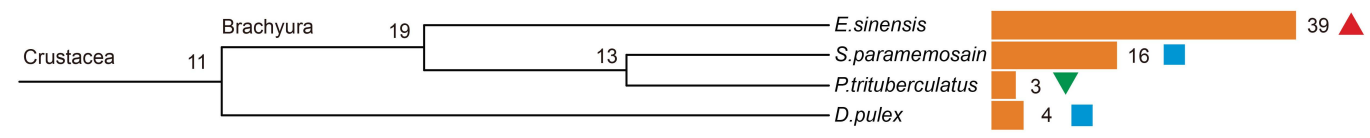

Figure 1. Distribution of DDE transposase 4 (DDE_Tnp_4) family genes gain and loss among crustaceans. The numbers on nodes represent a total number of DDE_Tnp_4 family genes for ancestral species. The red positive triangle represented that genes in this species expanded significantly. The green inverted triangle represented that genes in this species contracted significantly. The blue square represented that genes in this species did not expand or contract significantly.

Phylogenetic analysis revealed that DDE_Tnp_4 family genes could be categorized into four groups: I, II, III, and IV subfamilies (Figure 2). About 23 DDE_Tnp_4 family genes, including 14 from E. sinensis, 7 from S. paramamosain, and 4 from D. pulex, were clustered in subfamily I. A total of 18 DDE_Tnp_4 genes, including 17 from E. sinensis and 1 from $P$. trituberculatus, were clustered in subfamily II. Nine DDE_Tnp_4 genes, including seven from E. sinensis and two from S. paramamosain, were clustered in subfamily III. In the end, ten DDE_Tnp_4 genes, including one from E. sinensis, seven from S. paramamosain, and two from $P$. trituberculatus, were clustered in subfamily IV (Figure 2). The phylogenetic tree confirmed that DDE_Tnp_4 family was widely present in crustaceans, and there were certain differences in this family among different species. 


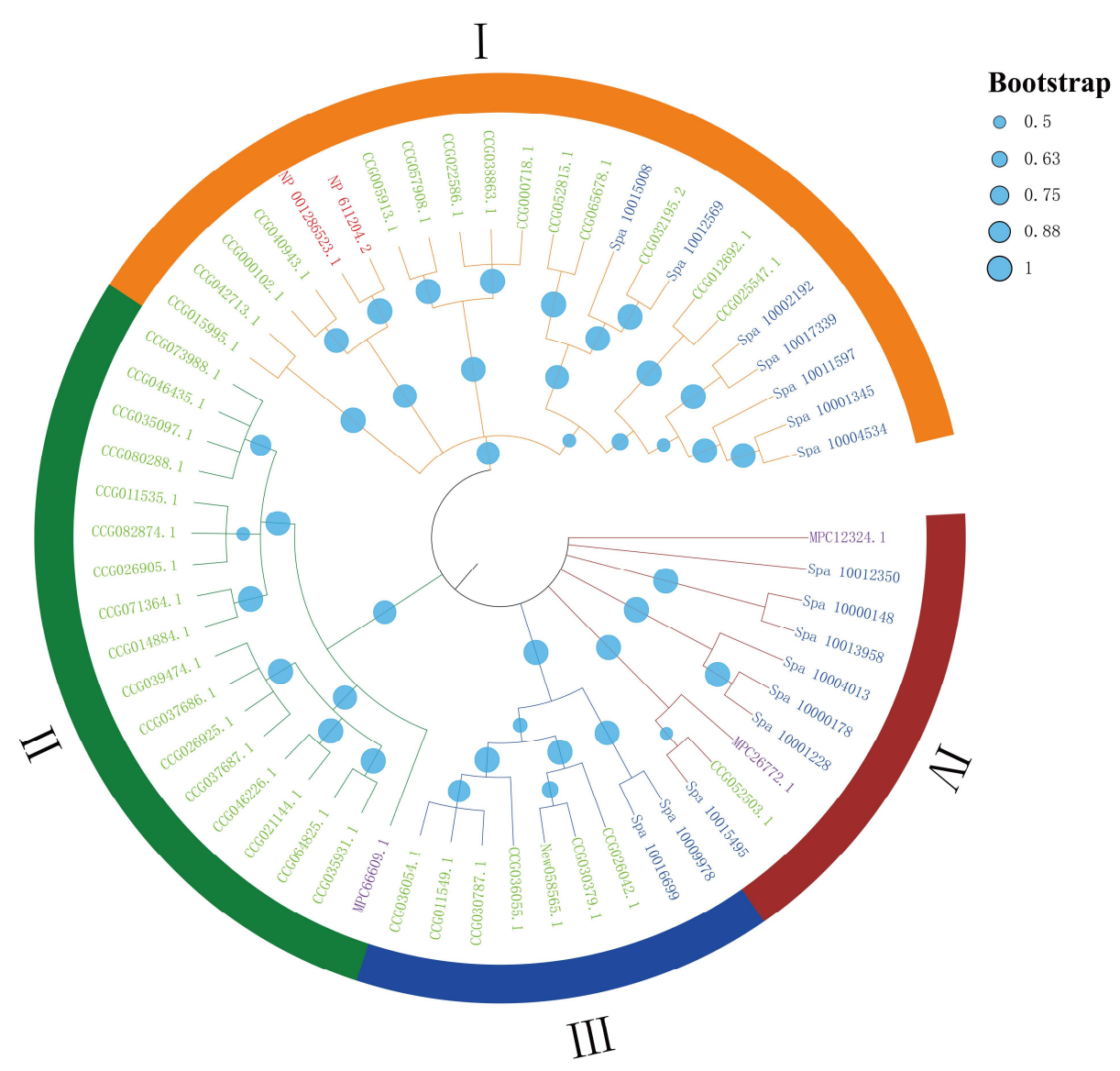

Figure 2. The phylogenetic tree of DDE_Tnp_4 family genes. Eriocheir sinensis, Scylla paramamosain, Portunus trituberculatus, and Daphnia pulex genes were distinguished by green, blue, purple, and red, respectively. Subfamily I, II, III, and IV were highlighted in orange, green, dark blue, and dark red, respectively.

\subsection{Conserved Motif and Gene Structure of DDE_Tnp_4 Family Genes in E. sinensis}

Multiple Em for Motif Elicitation (MEME) software was used to identify 15 conserved motifs among these E. sinensis DDE_Tnp_4 family genes. Each subfamily had its own motif distribution mode, and some motifs only appeared in a special subfamily (Figure 3). Motif 4 existed in all subfamily II genes and a part of subfamily I genes, Motif 1 was found in almost all subfamily II and III genes, Motif 6 was only present in a part of subfamily II genes, and Motif 15 was only found in subfamily III genes. This result indicated that different subfamilies had their own MEME characteristics, confirming the classification of the subfamilies of E. sinensis DDE_Tnp_4 family.

According to whole-genome gene annotations, the gene structure of E. sinensis DDE_Tnp_4 family genes was analyzed. Among 39 identified genes, $43.59 \%$ (17) of genes did not have introns, $33.33 \%$ (13) owned just one intron, $20.51 \%$ (8) possessed two introns, and only $2.56 \%$ (1) had three introns (Figure 4). In addition, lengths of these introns were found to exhibit polymorphism. Most lengths of these introns (93.94\%) varied from 100 to 3000 base pairs (bp). However, a part of introns $(6.06 \%)$ was quite long, exceeding $10,000 \mathrm{bp}$ in length, with the longest reaching 12,791 bp (one intron of CCG071364.1). 


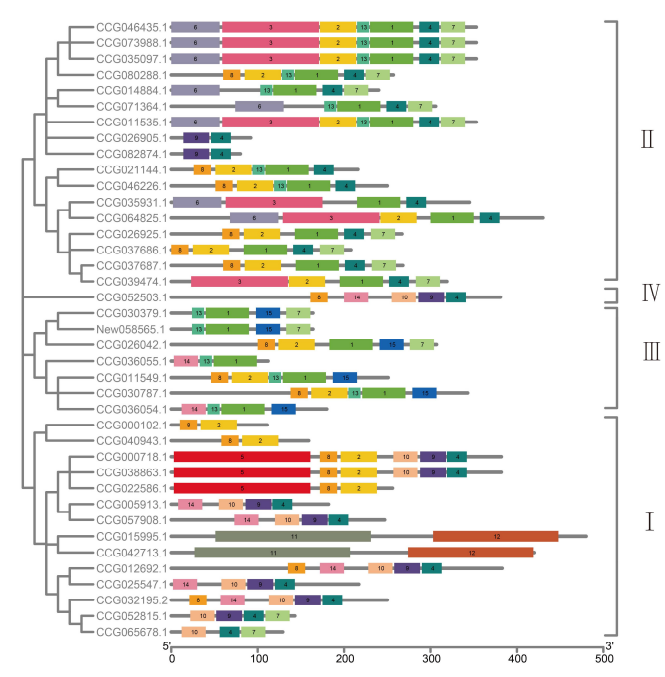

(a)

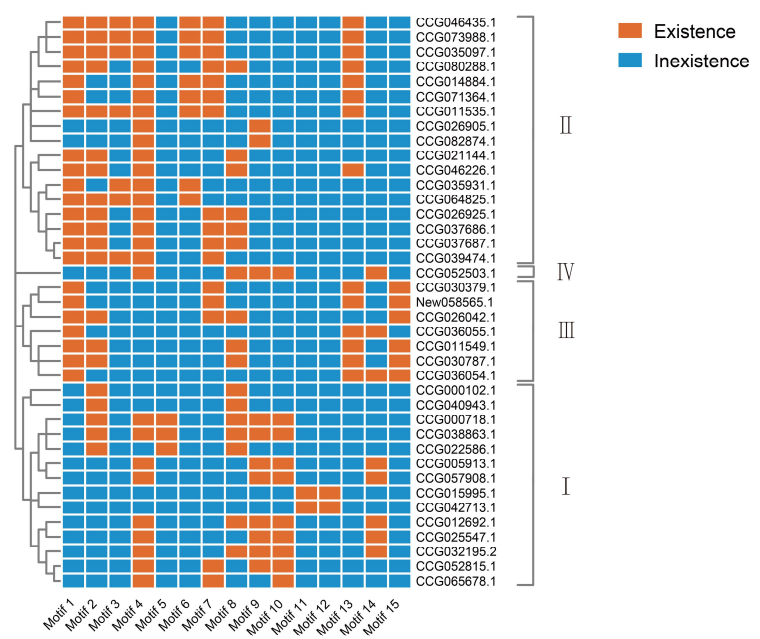

(b)

Figure 3. Conserved motifs of DDE_Tnp_4 family genes in E. sinensis. (a) The architecture of conserved protein motifs. (b) The heatmap indicates whether the meme motif existed in this gene. The orange modules imply that this gene contains this motif, while the blue ones do not.

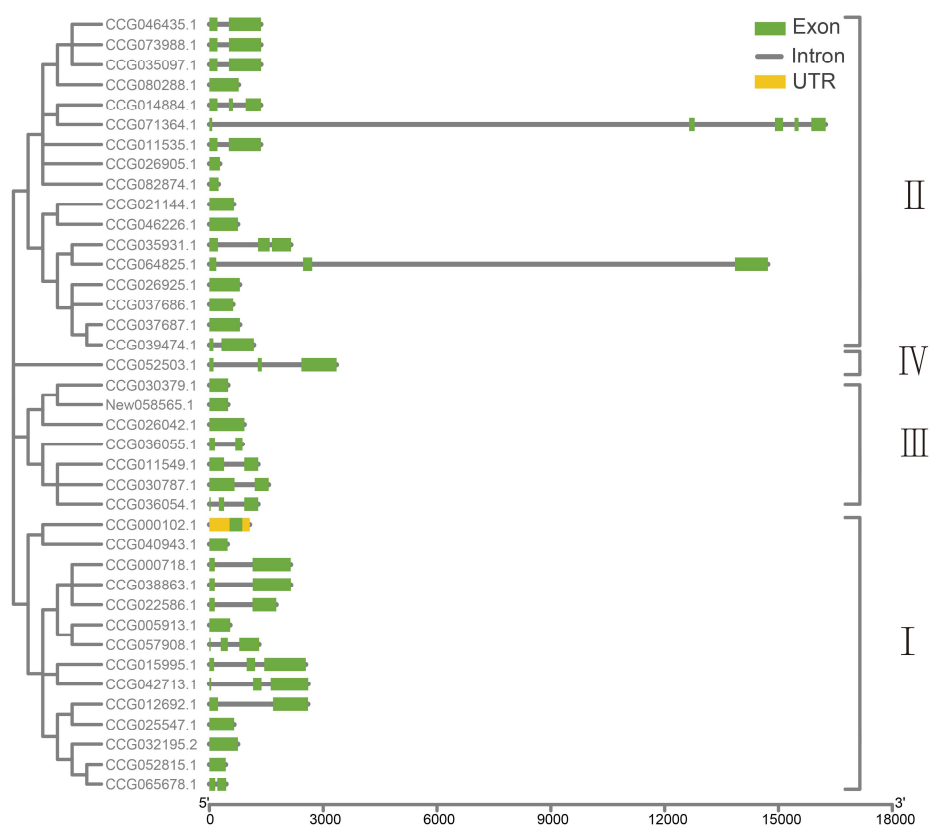

Figure 4. Gene structures of DDE_Tnp_4 family genes in E. sinensis. The green models mean exons, yellow models mean untranslated regions (UTR), and grey lines mean introns.

\subsection{Synteny and Duplication of DDE_Tnp_4 Family Genes in E. sinensis}

In total, 39 DDE_Tnp_4 family genes were mapped to 28 chromosomes of E. sinensis genome, and each chromosome contained 1-3 DDE_Tnp_4 family genes (Supplementary Materials). However, there were three couples of genes: (CCG011549.1 and CCG011535.1, CCG036054.1 and CCG036055.1, CCG037687.1 and CCG037686.1) tightly linked to each other, and their protein sequences were relatively conserved (Figure 5). 


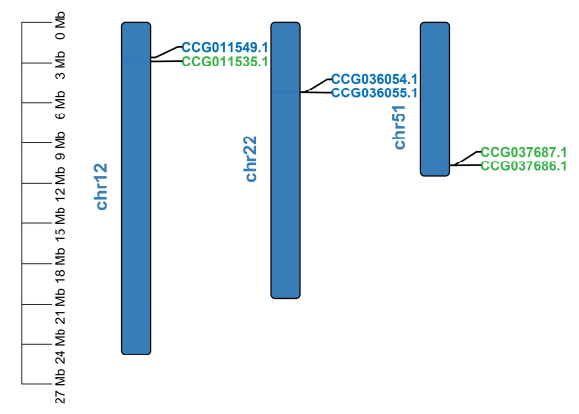

(a)

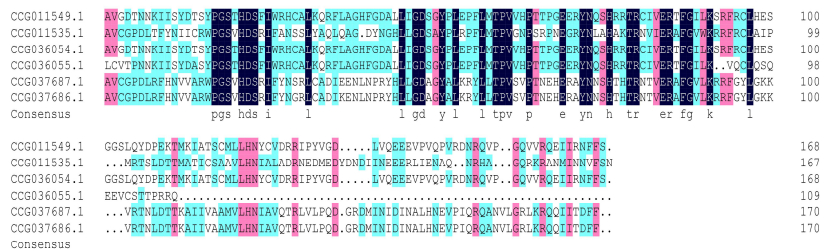

(b)

Figure 5. Tightly linked DDE_Tnp_4 family genes in E. sinensis. (a) Distribution of these genes identified in E. sinensis chromosomes. Subfamily II and III genes were distinguished by green and blue, respectively. (b) Multiple alignments of protein sequences. The dark blue shading indicates identical amino acid residues. Pink shading indicates less conserved residues. Light blue shading indicates somewhat similar residues.

\subsection{Gene Expression of E. sinensis DDE_Tnp_4 Family Genes under Acute High Salinity Stress and Air Exposure Stress}

To investigate the potential function of E. sinensis DDE_Tnp_4 family genes in coping with environmental stress, E. sinensis transcriptomes under acute high salinity and air exposure stress conditions were analyzed, the details of expression analysis, clustering analysis, and normalization information can be found in Figures S1-S4 in Supplementary Materials. Under acute high salinity stress conditions, 14 DDE_Tnp_4 genes were found to be expressed in E. sinensis hemocytes, among which 8, 4, 1, and 1 genes belong to subfamily I, II, III, and IV, respectively. Under air exposure stress conditions, 12 DDE_Tnp_4 genes were found to be expressed in gills of E. sinensis, among which 7, 3, 1, and 1 genes belong to subfamily I, II, III, and IV, respectively.

Under acute high salinity stress conditions, the expression of subfamily I genes showed largely high expression compared to control groups, while subfamily II genes were also up-regulated. Under air exposure stress conditions, subfamily I, II, and IV genes were generally up-regulated (Figure 6). This result indicated that DDE_Tnp_4 family might be involved in high salinity and air exposure stress conditions in E. sinensis.

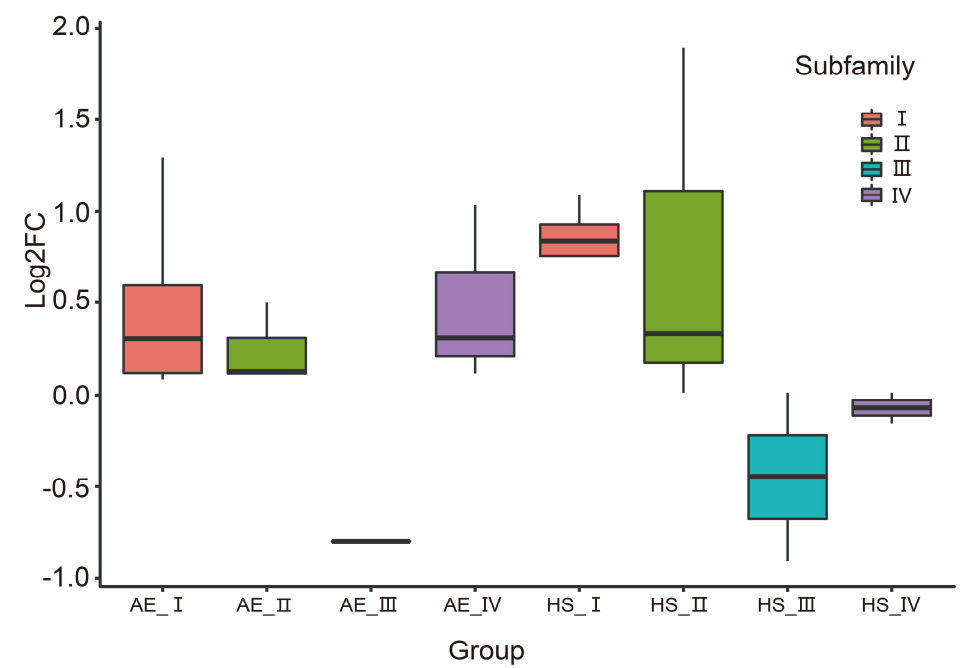

Figure 6. Global expression profiles of DDE_Tnp_4 family genes in E. sinensis hemocytes under acute high salinity conditions and in E. sinensis gills under air exposure stress conditions. AE_I-AE_IV represents respective subfamily genes under air exposure stress conditions. HS_I-HS_IV represents respective subfamily genes under acute high salinity stress conditions. 


\section{Discussion}

DDE_Tnp_4 is a large family containing numerous members which function in angiogenesis, cell cycle regulation, and innate immunity [18-20]. Herein, we identified 39 DDE_Tnp_4 family genes in E. sinensis genome, which are dispersedly distributed across 28 chromosomes. This phenomenon is consistent with the expansion of transposon-derived Iris genes in Drosophila melanogaster through "cut and paste" mechanism [23]. However, three couples of subfamilies II and III genes were found to be tightly linked to each other. According to judgment standards of tandem genes [24], these three couples of genes could be identified as tandem genes and resulted from tandem duplication, similar to numerous TLR genes found in Strongylocentrotus purpuratus [25]. Therefore, we speculate that DDE_Tnp_4 family genes in E. sinensis may be formed by various mechanisms such as "cut and paste" mechanism and tandem duplication of transposable elements. The number of introns in E. sinensis DDE_Tnp_4 family genes is relatively small; $43.59 \%$ (17) of genes lacked having introns, while $33.33 \%$ (13) of genes owned just one intron. This result is similar to the situation found in Danio rerio, where DDE_Tnp_4 family genes found in D. rerio lacked or possessed only one intron [26].

Transposons are a significant basic source of gene expansion. During species evolution, transposons replicate, move, amplify, and accumulate in invaded genomes [27]. Following molecular domestication, these inserted transposons become novel functional genes in host genomes [27]. To determine whether DDE_Tnp_4 family genes in E. sinensis have expanded, the genomic information of E. sinensis was compared with three other crustaceans. It was discovered that $E$. sinensis had a greater number and ratio of DDE_Tnp_4 family genes than S. paramamosain, P. trituberculatus, and D. pulex. Furthermore, expansion and contraction analyses confirmed that DDE_Tnp_4 family in E. sinensis genome expanded compared with other Brachyura species. The above findings are consistent with those reported for L. chalumnae; the expansion of DDE_Tnp_4 family genes is observed, and this phenomenon is speculated to be linked to adaptation of aquatic organisms to terrestrial environment [21]. Expansion of DDE_Tnp_4 family genes is also observed in A. pisum, and these expansive genes might have a significant role in the diversification of morphology and adaptations [22].

During gene expansion, the positive selection pressure promotes species to form more novel functional genes, which is more conducive for species to adapt to various biological or abiotic stresses [13]. For instance, $\mathrm{Cu} / \mathrm{Zn} \mathrm{SOD}$ family genes in $\mathrm{C}$. farreri have expanded significantly, which protects the body against the damaging effects from PSTs [14]. There are gene expansion phenomena among subfamilies $\mathrm{G}$ and $\mathrm{H}$ of $\mathrm{ABC}$ family in D. pulex, and these genes are involved in pollutant efflux and cell defense activities [15]. E. sinensis has complicated life history and high tolerance to environmental stress [3]. To verify whether excellent environmental adaptability of E. sinensis is linked to expansive DDE_Tnp_4 family genes, we analyzed expression profiles of E. sinensis DDE_Tnp_4 family genes under acute high salinity and air exposure stress conditions. Most DDE_Tnp_4 family genes were generally up-regulated under acute high salinity and air exposure stress conditions. The gene expression of subfamily I under high salt stress conditions was largely upregulated compared to the control group. The aforementioned findings indicated that DDE_Tnp_4 family might be involved in high salinity and air exposure stress. These results are consistent with those obtained in Marsupenaeus japonicus, where the expression of DDE_Tnp_4 family genes was significantly up-regulated when challenged with white spot syndrome virus (WSSV) [20].

According to the above results, it is speculated that expensive DDE_Tnp_4 family genes may be linked to environmental tolerance of E. sinensis. In addition, E. sinensis could adapt to abiotic stresses by activating innate immune system and many other physiological activities [8,9]. High salinity and air exposure stress have been demonstrated to activate TLR pathway and antioxidant response in E. sinensis [10-12]. As transcriptional regulators, DDE_Tnp_4 family is considered to specifically regulate transcription of target genes [26]. For instance, DDE_Tnp_4 family genes participate in TLR pathway by positively regulating 
Toll genes expression in M. japonicus [20]. As a result, we speculated that DDE_Tnp_4 family genes might participate in E. sinensis adaptation to environmental stress by regulating the transcription of target genes in immune system and physiological activities.

\section{Materials and Methods}

\subsection{Identification of DDE_Tnp_4 Family Genes}

The E. sinensis genome and annotation data were obtained from our previous study (accession number: LQIF00000000). The genome data of S. paramamosain, P. trituberculatus, and D. pulex were downloaded from the National Center for Biotechnology Information (NCBI) database (accession number: SRR12442560, SRR12442561, GCA_008373055.1, and GCA_000187875.1). To identify DDE_Tnp_4 family genes, Hidden Markov Model (HMM) profile of DDE_Tnp_4 family genes (accession number: PF13359) was downloaded from Pfam database [28], and a set of proteins from the genome of E. sinensis was scanned using hmmsearch from HMMER v3.3 suite [29] using HMM with an expected value threshold $<10^{-4}$ [30]. The results were further confirmed using the online tool SMART [31] (http:/ / smart.embl.de/, accessed on 8 October 2021). DDE_Tnp_4 family genes of S. paramamosain, P. trituberculatus, and D. pulex were also identified using the same methods.

\subsection{Gene Family Expansion and Contraction Analysis}

OrthoFinder [32] was used to identify single copy orthologue genes among genome data of E. sinensis, S. paramamosain, P. trituberculatus, and D. pulex. MEGA-X program was employed to construct the ultrametric tree according to single copy orthologue genes and fossil information from Fossil Calibration Database [33,34]. The numbers of DDE_Tnp_4 family genes and the ultrametric tree were input into CAFE to analyze the expansion and contraction situation. The output result was visualized using Interactive Tree of Life (iTol) [35] (https:/ / itol.embl.de/, accessed on 8 October 2021).

\subsection{Gene Family Expansion and Contraction Analysis}

Multiple sequence alignments were performed using MUSCLE tool [36]. A Maximum Likelihood (ML) phylogenetic tree was constructed by MEGA-X program using General Time Reversible (GTR) model with 1000 bootstrap replicates [33], and iTol [35] (https: //itol.embl.de/, accessed on 8 October 2021) was used to display the phylogenetic tree generated from MEGA-X program.

\subsection{Localization and Synteny Analysis of DDE_Tnp_4 Family Genes}

Location and synteny information of DDE_Tnp_4 family genes were obtained from E. sinensis genome annotations. Gene Location Visualize (Advanced) [37] was used to display DDE_Tnp_4 family genes on E. sinensis chromosomes. Tandem genes in E. sinensis were identified as previously reported standards [24]. Multiple sequence alignment of proteins was performed using CLUSTALW program packaged in DNAMAN 8.0 software.

\subsection{Localization and Synteny Analysis of DDE_Tnp_4 Family Genes}

MEME 5.1.1 [38] (http:/ / meme-suite.org/tools/meme, accessed on 28 September 2021) was used to scan conserved motifs. Parameters in MEME were as follows: the number of motifs, 15; minimum width, 6; maximum width, 200; other parameters were left at their default values. The global perspective of motifs in each DDE_Tnp_4 family genes was conducted by HeatMap [37]. The Gene Structure View (Advanced) [37] was employed to display the gene exon-intron structure and domain coding regions within default parameters.

\subsection{Expression Profiling of DDE_Tnp_4 Family Genes under Stress}

Under stress conditions, the profiles of DDE_Tnp_4 family genes were analyzed using transcriptome data obtained from previous studies [5] and the European Nucleotide 
Archive (ENA) database. During acute high salinity stress experiment, healthy crab individuals were divided into four groups; one group in freshwater serves as the control, and other three groups were challenged with $16 \%, 28 \%$, and $35 \%$ saltwater, respectively. Following that, hemocytes were sampled for RNA extraction at $24 \mathrm{~h}$ postsalinity challenge. During the air exposure stress experiment, healthy crab individuals were cultured for 1,3 , and 5 days with constant $27^{\circ} \mathrm{C}, 95 \%$ humidity, $14 \mathrm{~h}$ light, $10 \mathrm{~h}$ dark, and no water. For the control group, ten crab individuals were cultured in water $\left(27^{\circ} \mathrm{C}\right)$. Then, gill tissues from each group were quickly collected and used to extract RNA. Raw data of transcriptome sequencing were downloaded from ENA database (accession number: SRR6516036, SRR6516037, SRR6516038, SRR6516039, SRR6516040, SRR6516041, SRR7507779, SRR7507780, SRR7507781, and SRR7507782). Quality control and filtering of raw reads were conducted using FastQC [39] and Trimmomatic [40] software, respectively. De novo transcriptome assembly was performed using Trinity 2.6.6 [41]. Expression abundance of transcript was calculated as transcripts per million mapped reads (TPM) value by using scripts in Trinity. The read counts were adjusted by edgeR program package through one scaling normalized factor, and differential expression analysis of two samples was performed using DEGseq R package [42]. The global perspective of DDE_Tnp_4 family genes expression level was conducted using ggplot2 R package [43].

Supplementary Materials: The following are available online at https:/ / www.mdpi.com/article/10. 3390/antibiotics10121430/s1. Figure S1: Conserved motifs of DDE_Tnp_4 family genes in E. sinensis, S. paramamosain, P. trituberculatus, and D. pulex. Figure S2: Global expression profiles of genes in E. sinensis hemocytes under acute high salinity conditions. Figure S3: Global expression profiles of genes in E. sinensis gills under acute air exposure stress conditions. Figure S4: The normalization and distribution of gene expression in E. sinensis hemocytes under acute high salinity conditions. Sequence S1: Amino acid sequences of DDE_Tnp_4 family in E. sinensis, S. paramamosain, P. trituberculatus, and D. pulex.

Author Contributions: Conceptualization, Y.X., Y.Y. and J.Z.; methodology, Y.X.; software, Y.X.; validation, Y.X., Y.Y., J.Z. and Z.C.; formal analysis, Y.X., Y.Y., J.Z. and Z.C.; investigation, Y.X. and Y.Y.; resources, Y.X.; data curation, Y.X. and Y.Y.; writing-original draft preparation, Y.X.; writing-review and editing, Y.X., Y.Y., J.Z. and Z.C.; visualization, Y.X.; supervision, Z.C.; project administration, Z.C.; funding acquisition, Z.C. All authors have read and agreed to the published version of the manuscript.

Funding: This research was funded by grants from the National Key R\&D Program of China (2018YFD0900303), National Natural Science Foundation of China (32072964) and the Ten Thousand Talents Program.

Institutional Review Board Statement: Not applicable.

Informed Consent Statement: Not applicable.

Data Availability Statement: The data presented in this study are available in Supplementary Material.

Conflicts of Interest: The authors declare no conflict of interest.

\section{References}

1. Liu, L.; Cui, Z.; Song, C.; Liu, Y.; Hui, M.; Wang, C. Flow cytometric analysis of DNA content for four commercially important crabs in China. Acta Oceanol. Sinica 2016, 35, 7-11. [CrossRef]

2. Herborg, L.M.; Rushton, S.P.; Clare, A.S.; Bentley, M.G. The invasion of the Chinese mitten crab (Eriocheir sinensis) in the United Kingdom and its comparison to continental Europe. Biol. Invasions 2005, 7, 959-968. [CrossRef]

3. Dittel, A.I.; Epifanio, C.E. Invasion biology of the Chinese mitten crab Eriocheir sinensis: A brief review. J. Exp. Mar. Biol. Ecol. 2009, 374, 79-92. [CrossRef]

4. Long, X.; Wu, X.; Zhao, L.; Ye, H.; Cheng, Y.; Zeng, C. Effects of salinity on gonadal development, osmoregulation and metabolism of adult male Chinese mitten crab, Eriocheir sinensis. PLoS ONE 2017, 12, e0179036. [CrossRef] [PubMed]

5. Chen, X.; Wang, J.; Hou, X.; Yue, W.; Li, Z.; Wang, C. Gene expression profiles of gill provide insights into the aerial respiration capacity of the Chinese mitten crab, Eriocheir sinensis. Aquaculture 2019, 506, 148-153. [CrossRef]

6. Lu, Y.; Zhang, D.; Wang, F.; Dong, S. Hypothermal effects on survival, energy homeostasis and expression of energy-related genes of swimming crabs Portunus trituberculatus during air exposure. J. Therm. Biol. 2016, 60, 33-40. [CrossRef] [PubMed] 
7. Lv, J.; Liu, P.; Wang, Y.; Gao, B.; Chen, P.; Li, J. Transcriptome analysis of Portunus trituberculatus in response to salinity stress provides insights into the molecular basis of osmoregulation. PLOS ONE 2013, 8, e82155.

8. Long, X.; Wu, X.; Zhao, L.; Ye, H.; Cheng, Y.; Zeng, C. Physiological responses and ovarian development of female Chinese mitten crab Eriocheir sinensis subjected to different salinity conditions. Front. Physiol. 2018, 8, 1072. [CrossRef] [PubMed]

9. Cheng, C.H.; Ma, H.L.; Deng, Y.Q.; Feng, J.; Chen, X.L.; Guo, Z.X. Transcriptome analysis and histopathology of the mud crab (Scylla paramamosain) after air exposure. Comp. Biochem. Physiol. C Toxicol. Pharmacol. 2020, 228, 108652. [CrossRef]

10. Li, E.; Wang, S.; Li, C.; Wang, X.; Chen, K.; Chen, L. Transcriptome sequencing revealed the genes and pathways involved in salinity stress of Chinese mitten crab, Eriocheir sinensis. Physiol. Genomics 2014, 46, 177-190. [CrossRef]

11. Sun, M.; Jiang, K.; Zhang, F.; Zhang, D.; Shen, A.; Jiang, M.; Shen, X.; Ma, L. Effects of various salinities on Na(+)-K(+)-ATPase, Hsp70 and Hsp90 expression profiles in juvenile mitten crabs, Eriocheir sinensis. Genet. Mol. Res. 2012, 11, 978-986. [CrossRef]

12. Bao, J.; Xing, Y.N.; Jiang, H.B.; Li, X.D. Identification of immune-related genes in gills of Chinese mitten crabs (Eriocheir sinensis) during adaptation to air exposure stress. Fish. Shellfish Immunol. 2019, 84, 885-893. [CrossRef] [PubMed]

13. Zheng, Y.; Wang, L.B.; Sun, S.F.; Liu, S.Y.; Liu, M.J.; Lin, J. Phylogenetic and ion-response analyses reveal a relationship between gene expansion and functional divergence in the $\mathrm{Ca}^{2+} /$ cation antiporter family in Angiosperms. Plant. Mol. Biol. 2021, 105, 303-320. [CrossRef] [PubMed]

14. Lian, S.; Zhao, L.; Xun, X.; Lou, J.; Li, M.; Li, X.; Wang, S.; Zhang, L.; Hu, X.; Bao, Z. Genome-Wide Identification and Characterization of SODs in Zhikong Scallop Reveals Gene Expansion and Regulation Divergence after Toxic Dinoflagellate Exposure. Mar. Drugs. 2019, 17, 700. [CrossRef] [PubMed]

15. Jeong, C.B.; Kim, H.S.; Kang, H.M.; Lee, J.S. ATP-binding cassette (ABC) proteins in aquatic invertebrates: Evolutionary significance and application in marine ecotoxicology. Aquat. Toxicol. 2017, 185, 29-39. [CrossRef]

16. Ru, H.; Mi, W.; Zhang, P.; Alt, F.W.; Schatz, D.G.; Liao, M.; Wu, H. DNA melting initiates the RAG catalytic pathway. Nat. Struct. Mol. Biol. 2018, 25, 732-742. [CrossRef]

17. Hickman, A.B.; Chandler, M.; Dyda, F. Integrating prokaryotes and eukaryotes: DNA transposases in light of structure. Crit. Rev. Biochem. Mol. Biol. 2010, 45, 50-69. [CrossRef] [PubMed]

18. Roussigne, M.; Kossida, S.; Lavigne, A.C.; Clouaire, T.; Ecochard, V.; Glories, A.; Amalric, F.; Girard, J.P. The THAP domain: A novel protein motif with similarity to the DNA-binding domain of P element transposase. Trends Biochem. Sci. 2003, 28, 66-69. [CrossRef]

19. Sabogal, A.; Lyubimov, A.Y.; Corn, J.E.; Berger, J.M.; Rio, D.C. THAP proteins target specific DNA sites through bipartite recognition of adjacent major and minor grooves. Nat. Struct. Mol. Biol. 2010, 17, 117-145. [CrossRef] [PubMed]

20. Sun, B.; Qian, X.; Zhu, F. Molecular characterization of shrimp harbinger transposase derived 1 (HARBI1)-like and its role in white spot syndrome virus and Vibrio alginolyticus infection. Fish Shellfish Immunol. 2018, 78, 222-232. [CrossRef] [PubMed]

21. Smith, J.J.; Sumiyama, K.; Amemiya, C.T. A Living Fossil in the Genome of a Living Fossil: Harbinger Transposons in the Coelacanth Genome. Mol. Biol. Evol. 2012, 29, 985-993. [CrossRef]

22. Vidal, N.M.; Grazziotin, A.L.; Iyer, L.M.; Aravind, L.; Venancio, T.M. Transcription factors, chromatin proteins and the diversification of Hemiptera. Insect Biochem. Mol. Biol. 2016, 69, 1-13. [CrossRef]

23. Malik, H.S.; Henikoff, S. Positive selection of Iris, a retroviral envelope-derived host gene in Drosophila melanogaster. PloS Genet. 2005, 1, 429-443. [CrossRef]

24. Wang, J.L.; Hu, T.H.; Wang, W.H.; Hu, H.J.; Wei, Q.Z.; Bao, C.L. Investigation of evolutionary and expressional relationships in the function of the leucine-rich repeat receptor-like protein kinase gene family (LRR-RLK) in the radish (Raphanus sativus L.). Sci. Rep. 2019, 9, 6937. [CrossRef] [PubMed]

25. Honoo, S.; Toshio, S. Toll-Like Receptors of Deuterostome Invertebrates. Front. Immunol. 2012, 3, 34.

26. Kapitonov, V.V.; Jurka, J. Harbinger transposons and an ancient HARBI1 gene derived from a transposase. DNA Cell Biol. 2004, 23, 311-324. [CrossRef] [PubMed]

27. Alzohairy, A.M.; Gyulai, G.; Jansen, R.K.; Bahieldin, A. Transposable elements domesticated and neofunctionalized by eukaryotic genomes. Plasmid 2013, 69, 1-15. [CrossRef] [PubMed]

28. Finn, R.D.; Tate, J.; Mistry, J.; Coggill, P.C.; Sammut, S.J.; Hotz, H.R.; Ceric, G.; Forslund, K.; Eddy, S.R.; Sonnhammer, E.L.; et al. The Pfam protein families database. Nucleic Acids Res. 2008, 36, D281-D288. [CrossRef]

29. Finn, R.D.; Clements, J.; Eddy, S.R. HMMER web server: Interactive sequence similarity searching. Nucleic Acids Res. 2011, 39, W29-W37. [CrossRef]

30. Wang, Y.C.; Lu, Q.H.; Xiong, F.; Hao, X.Y.; Wang, L.; Zheng, M.X.; Li, N.N.; Ding, C.Q.; Wang, X.C.; Yang, Y.J. Genomewide identification, characterization, and expression analysis of nucleotide-binding leucine-rich repeats gene family under environmental stresses in tea (Camellia sinensis). Genomics 2020, 112, 1351-1362. [CrossRef] [PubMed]

31. Schultz, J.; Milpetz, F.; Bork, P.; Ponting, C.P. SMART, a simple modular architecture research tool: Identification of signaling domains. Proc. Natl. Acad. Sci. USA 1998, 95, 5857-5864. [CrossRef]

32. Emms, D.M.; Kelly, S. OrthoFinder: Solving fundamental biases in whole genome comparisons dramatically improves orthogroup inference accuracy. Genome Biol. 2015, 16, 157. [CrossRef]

33. Kumar, S.; Stecher, G.; Li, M.; Knyaz, C.; Tamura, K. MEGA X: Molecular Evolutionary Genetics Analysis across Computing Platforms. Mol. Biol. Evol. 2018, 35, 1547-1549. [CrossRef] 
34. Ksepka, D.T.; Parham, J.F.; Allman, J.F.; Benton, M.J.; Carrano, M.T.; Cranston, K.A.; Donoghue, P.C.; Head, J.J.; Hermsen, E.J.; Irmis, R.B.; et al. The Fossil Calibration Database-A New Resource for Divergence Dating. Syst. Biol. 2015, 64, 853-859. [CrossRef]

35. Letunic, I.; Bork, P. Interactive Tree Of Life (iTOL): An online tool for phylogenetic tree display and annotation. Bioinformatics 2007, 23, 127-128. [CrossRef]

36. Waterhouse, A.M.; Procter, J.B.; Martin, D.M.; Clamp, M.; Barton, G.J. Jalview Version 2-a multiple sequence alignment editor and analysis workbench. Bioinformatics 2009, 25, 1189-1191. [CrossRef]

37. Chen, C.; Chen, H.; Zhang, Y.; Thomas, H.R.; Frank, M.H.; He, Y.; Xia, R. TBtools-an integrative toolkit developed for interactive analyses of big biological data. Mol. Plant. 2020, 8, 1194-1202. [CrossRef]

38. Bailey, T.L.; Boden, M.; Buske, F.A.; Frith, M.; Grant, C.E.; Clementi, L.; Ren, J.; Li, W.W.; Noble, W.S. MEME SUITE: Tools for motif discovery and searching. Nucleic Acids Res. 2009, 37, W202-W208. [CrossRef]

39. Brown, J.; Pirrung, M.; Mccue, L.A. FQC Dashboard: Integrates FastQC results into a web-based, interactive, and extensible FASTQ quality control tool. Bioinformatics 2017, 33, 3137-3139. [CrossRef]

40. Bolger, A.M.; Lohse, M.; Usadel, B. Trimmomatic: A flexible trimmer for Illumina sequence data. Bioinformatics 2014, 30, 2114-2120. [CrossRef]

41. Haas, B.J.; Papanicolaou, A.; Yassour, M.; Grabherr, M.; Blood, P.D.; Bowden, J.; Couger, M.B.; Eccles, D.; Li, B.; Lieber, M.; et al. De novo transcript sequence reconstruction from RNA-seq using the Trinity platform for reference generation and analysis. Nat. Protoc. 2013, 8, 1494-1512. [CrossRef]

42. Robinson, M.D.; Mccarthy, D.J.; Smyth, G.K. edgeR: A Bioconductor package for differential expression analysis of digital gene expression data. Bioinformatics 2010, 26, 139-140. [CrossRef]

43. Ginestet, C. ggplot2: Elegant Graphics for Data Analysis. J. R. Stat. Soc. A Stat. 2011, 174, 245. [CrossRef] 